\title{
APUNTES DE LA GESTACIÓN SUBROGADA
}

\author{
NOTES OF THE SURROGATE GESTATION
}

\author{
Carmita Sánchez-Madrigal ${ }^{1} *$ (D). \\ 1. Escuela Judicial del Estado de Tabasco, México. carmitaprimerocivil@hotmail.com \\ * Autor de correspondencia: Carmita Sánchez-Madrigal, correo electrónico: carmitaprimerocivil@hotmail.com
}

\section{RESUMEN}

La gestación subrogada en un tema controversial con diversas aristas, este documento se basa en el acercamiento y descripción de la gestión subrogada altruista y remunerada. Por lo cual deja abierta la línea de investigación en el derecho de las partes involucradas (padre contratante, mujer gestante y el menor).

Describe los Estados de la República Mexicana que consideran la gestación subrogada señalando alcances y factores que intervienen como la moral, la religión, el aspecto político y legislativo a partir del reconocimiento y garantía de los derechos humanos en materia de género en específico de la mujer y el interés superior del menor. Finalmente, basado en un proceso interpretativo y analítico los apuntes de la de gestión subrogada ofrece al lector un criterio y perspectiva de los alcances de este proceso dentro del ámbito del derecho.

Palabras clave: Derecho civil; familia; interés superior del menor; maternidad.

Cómo citar:

Sánchez-Madrigal, Carmita. (2021). Apuntes de la gestación subrogada. Revista de Investigaciones

Universidad del Quindio, 33(S2), 49-54. https://doi.org/10.33975/riuq.vol33nS2.611 


\begin{abstract}
Surrogacy in a controversial issue with various edges, this document is based on the approach and description of altruistic and remunerated surrogacy management. Therefore, it leaves open the line of investigation in the law of the parties involved (contracting father, pregnant woman and the minor). Describes the States of the Mexican Republic that consider surrogacy, pointing out the scope and factors that intervene such as morality, religion, the political and legislative aspect based on the recognition and guarantee of human rights in gender matters, specifically of women and the best interests of the minor. Finally, based on an interpretive and analytical process, the notes of the surrogate management offer the reader a criterion and perspective of the scope of this process within the scope of law.
\end{abstract}

Keywords: Civil law; family; best interests of the minor; maternity.

\title{
INTRODUCCIÓN
}

La gestación subrogada es un contrato a través del cual una mujer acepta gestar para una persona o pareja que tienen la intención de ser padres o madre, del niño o niña nacido de dicho embarazo (Briceño \& Jurado, 2018).

Coleman describe la maternidad subrogada como:

"una aplicación novel de la técnica de la inseminación artificial que resulta en el nacimiento de una criatura con un nexo biológico unilateral a la pareja infértil. La gestante es una mujer fértil que conviene que, mediante contrato, se la insemine artificialmente con el semen de un hombre casado con otra mujer, gestar el niño y darla a luz o procrearla. Una vez nacido el niño, la gestante o suplente renuncia su custodia a favor del padre biológico y, además, termina todos sus derechos de filiación sobre el niño para que la esposa del hombre con cuyo semen fue inseminada la adopte" (Coleman, 1982, p. 75).

La gestación por contrato admite las siguientes modalidades:

- Gestación subrogada: implica que la gestante sea inseminada aportando sus propios óvulos y que después del parto entregue al recién nacido a la madre o padres sus contratantes, a través de la adopción plena.

- Gestación sustituta: implica que la gestante sea contratada exclusivamente para aportar en su vientre al embrión obtenido por la fecundación de gametos de la pareja o persona contratante.

Existe otro tipo de diferenciación en relación a la gestación subrogada, ya que esta puede ser de manera altruista o remunerada.

La primera se da cuando la mujer lo hace sin ánimo de lucro. No obstante, los padres biológicos se responsabilizan de los gastos médicos y legales.

La segunda se da cuando la madre gestante acepta a cambio de un monto económico. 
En este proceso de relación es importante tener conocimiento respecto al alcance normativo y la propia administración de justicia, en caso de acontecer una situación de conflicto de interés entre las partes poder tener acceso a un proceso que permita determinar dentro del ámbito jurídico las determinantes del caso.

\section{METODOLOGÍA}

La gestación subrogada tiene muchos matices que requieren ser analizados, desde la óptica de los padres contratantes, la madre gestante e incluso del propio menor.

En este caso se abordará desde el punto de vista de la madre gestante en relación a si la gestación subrogada debe de ser únicamente altruista o remunerada, dado que de los países que contemplan esta figura, algunos solo la permiten de manera altruista. Para el caso mexicano, el estado de Tabasco, tiene reglamentada la gestión subrogada en el Código Civil del Estado (1997).

Enlazado con lo anterior, también se requiere analizar si es considerada cómo ética o no la conducta de la madre gestante que opta por recibir una remuneración, ello con independencia de los gastos médicos que deben erogar los padres contratantes durante el parto e incluso posterior a él.

Por lo que se aborda desde un alcance de comprensión, análisis e interpretación jurídica como el método axiológico dando pauta a un proceso de comprensión de hecho social adherido a una manifestación de comprensión desde el ámbito del derecho.

\section{RESULTADOS}

\section{Antecedentes institucionales}

La gestación subrogada, desde sus inicios ha sido un tema muy controvertido, fue en Estados Unidos en el año de 1985, donde se dio el primer caso documentado sobre la gestación subrogada (Albornoz \& Velarde, 2020). Suscitándose por vez primera transferir al útero de una mujer, un embrión creado con los gametos de los padres, mediante la técnica de fecundación in vitro- FIV "hasta los años 90 la modalidad de gestación subrogada utilizada era la denominada completa, ya que la mujer gestante también aportaba su óvulo. De los años 90 en adelante, con la popularización de la FIV, la modalidad utilizada es la conocida como parcial: el óvulo que se fecunda pertenece a una mujer diferente a la gestante, es decir, proviene de una proveedora o de la madre de intención, si la hay y es posible" (Moreno, 2018).

En 1980 se firmó el primer acuerdo comercial de gestación subrogada, que permitió a la gestante Elizabeth Kane recibir 10,000.00 dólares por dar a la luz al bebé de otra pareja (Zapata, 2019).

En 1986 el caso conocido como Baby M fue decisivo en la historia de la gestación subrogada, "desde entonces, la gestación por sustitución se ha convertido en un método cada vez más popular en lo concerniente a la tecnología reproductiva, aunque su aceptación no es pacífica” (Lamn, 2013).

En México, solo dos entidades federativas permiten este tipo de contratos dentro de su normativa los cuales son los estados de Sinaloa y Tabasco. 
En 1997 el Estado de Tabasco introdujo una regulación sobre la gestación subrogada en su Código Civil, que simplemente contemplaba el registro de menores nacidos a partir de estos acuerdos (Tosca, 2016).

En ambos estados las legislaciones atienden a temas de orden civil, sin embargo, la regulación de la gestación subrogada implica cuestiones sanitarias, que son materia de salubridad en general y, por tanto, competencia de la federación.

"Dado que los acuerdos de la gestación subrogada utilizan técnicas de reproducción asistida (TRA), por el establecimiento del embarazo, la ausencia de una regulación sobre reproducción asistida en México afecta las condiciones en que se llevan a cabo estos contratos" (Grupo de Información en Reproducción Elegida, 2017).

En los países la postura del gobierno acerca de la gestación subrogada es muy diversa, desde la prohibición hasta la autorización (Pérez, 2018), incluso toleran la ausencia de un marco legal explícito. La gestación subrogada se considera hoy en día una técnica de reproducción asistida compleja. Si no se aplica dentro de un marco legal apropiado y en adecuación con criterios éticos, puede dar paso a situaciones de abuso o ser contraria a los respetos de los Derechos Humanos.

\section{Desarrollo de los argumentos en favor y en contra.}

Normalmente las parejas que desean tener un hijo de manera natural y que no logran una gestación, se someten a múltiples tratamientos de fertilidad, de no lograr resultados positivos optan por la gestación subrogada, que es la única forma de tener un hijo y así hacer su sueño realidad, éste método también es útil para las mujeres solteras y las parejas homosexuales.

La gestación subrogada está permitida en tan sólo unos pocos países del mundo, y es legal únicamente en aquellos países donde existe una ley que permite expresamente esta práctica reproductiva.

Aunque las legislaciones varían en cada país, generalmente indican las condiciones o requisitos para poder llevarla a cabo, incluyendo aspectos como de aquellas personas que pueden realizar la técnica, como se establece la filiación del menor (Valdés, 2014) y los derechos y obligaciones que los padres intencionales y la gestante.

La gestación subrogada es "una práctica controversial y compleja, que implica considerar diferentes dimensiones de análisis, como las posibles desavenencias entre las partes, la discusión sobre si la gestación subrogada debe ser remunerada o altruista, la situación de quienes deben tener acceso a los contratos entre otros” (Grupo de Información en Reproducción Elegida, 2017).

Dado los múltiples cuestionamientos, aquí sólo se analizará el tema de si la gestación subrogada debe ser remunerada o altruista, y de ser remunerada, si la madre gestante está actuando de una manera no ética o si por el contrario esto garantiza la protección de sus derechos humanos.

\section{Sustento de los argumentos}

La remuneración económica es uno de los elementos más controversiales en la discusión sobre la gestación subrogada, por lo cual se dan opiniones encontradas al respecto (Olavarría, 2018). 
Por un lado, se presentan aquellos que señalan que la gestación subrogada debe ser únicamente de manera altruista, ya que de aceptar la madre una remuneración, estaría comercializando con el producto lo cual consideran no ético, en razón de que un hijo ni se compra ni se vende.

Desde otro punto de vista o posición están los que sostienen que la gestación subrogada no solo debe ser de manera altruista sino también remunerada (Bellver, 2017), partiendo de que la mujer tiene derecho a decidir sobre su cuerpo, lo que conlleva al respeto de sus derechos humanos, incluso argumentan que si en una relación de patrón a trabajador hay forzosamente una remuneración, equiparando dicha relación con la gestación subrogada (Cámara de Diputados, 2008), donde se puede considerar a los padres contratantes como patrón y a la madre gestante como el trabajador que presta un servicio, debe también haber una remuneración.

Sin embargo, este documento apunta a que la gestación subrogada no debe de ser únicamente altruista, pues aun cuando se establece en los códigos de algunos países, ello es una falacia puesto que la realidad es otra, tampoco puede considerarse como no ética la conducta de la madre gestante por optar en recibir una remuneración.

Se comparte la postura de que la gestación subrogada debe ser remunerada, pues además de comulgar con las razones que la sustentan, la remuneración es justa, si se toma en cuenta todos los malestares por los que tiene que pasar una mujer en su embarazo, tales como nauseas, mareos, presión alta o baja, y existen casos en que se dan embarazos de alto riesgo en donde la madre debe permanecer en absoluto reposo, teniendo cambios no sólo físicos sino en su proceso de vida cotidiana.

Se considera el alcance en el momento del parto etapa en donde la madre corre un riesgo latente por las complicaciones que pudieran presentarse e incluso la muerte, luego entonces, estas son razones más que suficientes para considerar que la gestación subrogada debe de ser remunerada. Es de resaltar que en caso de que la madre gestante opte por que la gestación subrogada sea remunerada, de ninguna manera puede considerarse su actuar como una conducta no ética, puesto que moralmente no hay nada que le impida hacerlo, por el contrario, es un derecho que le asiste.

\section{DISCUSIÓN}

Desde sus inicios el tema de la gestación subrogada ha sido muy complejo su discusión en el plano nacional e internacional respecto a derechos reproductivos, adquieren cada vez mayor importancia. Son pocos los países que la permiten, y su regulación varia conforme a la normativa y legislación de cada nación.

En el caso de México solo dos estados la permiten Sinaloa y Tabasco, en sus regulaciones se advierten lagunas que requieren de un minucioso estudio, pues éstas deben tener como eje central la garantía de los derechos de las tres partes de un acuerdo de gestación subrogada: La de los padres contratantes o intencionales, la de las mujeres gestantes y el de los niños y niñas nacidos por estos acuerdos.

También deben garantizar dichas legislaciones la no discriminación, así como prototipos de género, para evitar las violaciones a los derechos humanos establecidos en la Constitución.

Al permitir algunas codificaciones la gestación subrogada sólo de manera altruista, sin dar opción a la madre gestante a recibir una remuneración, se considera una violación a sus derechos humanos, puesto 
que esta tiene derecho a decidir sobre su cuerpo. Por último, el hecho de permitir que la gestación subrogada sea además de altruista también remunerada, y que se codifique su pago, considera que conlleva beneficios a las partes contratantes, pues por una parte se terminaría con el mercado negro que existe y por otro se evitaría la explotación que se da a la mujer gestante al recibir un pago no adecuado, no justo, garantizándole con ello sus derechos.

\section{REFERENCIAS}

1. Albornoz, María Mercedes \& Velarde Méndez, Mónica María Antonieta. (2020). Aproximación a la gestación por sustitución. IIJUNAM-CIDE, México.

2. Bellver Capella, Vicente. (2017). Tomarse en serio la maternidad subrogada altruista. Cuadernos de bioética, XXVIII, 229-243.

3. Briseño Montes, Centella \& Jurado Parres, Hans. (2018). Gestación sustituta y subrogada en México, derecho humano no reconocido constitucionalmente. Derechos Fundamentales a Debate/ Comisión Estatal de Derechos Humanos Jalisco. Instituto de Investigación y Capacitación de Derechos Humanos.

4. Cámara de Diputados. (2008). Maternidad subrogada. Centro de Documentación, Información y Análisis Dirección de Servicios de Investigación y Análisis Subdirección de Política Exterior. México.

5. Coleman, Phyllis. (1982). Surrogate motherhood: analysis of the problems and suggestions for solutions. Tenessee Law Review, (50), 71-118.

6. Grupo de Información en Reproducción Elegida. (2017). Gestación subrogada en México. Resultados de una mala regulación. México.

7. Lamn, Eleonora. (2013). Gestación por sustitución. Ni maternidad subrogada ni alquiler de vientres. Universidad de Barcelona, España.

8. Morero Beltrán, Anna María. (2018). Características de las familias creadas por gestación subrogada en el Estado español. Papeles del CEIC. International Journal on Collective Identity Research, (2).

9. Olavarría, María Eugenia. (2018). La gestante sustituta en México y la noción de trabajo reproductivo. Revista interdisciplinaria de estudios de género de El Colegio de México, 4, e144. https://doi.org/10.24201/eg.v4i0.144

10. Pérez Hernández, Yolinliztli. (2018). Gestación subrogada: una revisión etnográfica para contribuir al debate en México. Debate feminista, 56, 85-109. Epub 20 de noviembre de 2020.https://doi. org/10.22201/cieg.2594066xe.2018.56.05

11. Periódico Oficial. (1997). Código Civil para el Estado de Tabasco. México.

12. Tosca, Ernesto Alonso. (2016). La maternidad subrogada y sustituta en el marco jurídico de Tabasco. Hechos y derechos, (33).

13. Valdés Díaz, Caridad del Carmen. (2014). La maternidad subrogada y los derechos de los menores nacidos mediante el uso de esas técnicas. Anuario de la Facultad de Derecho, vol. XXXI, 459-482.

14. Zapata Denis, María Minerva. (2019). La maternidad subrogada en México. Tohil Revista Jurídica de la Facultad de Derecho, 43(I). 